\title{
Neutrino Physics Today, Important Issues and the Future
}

Stephen J. Parke

Theoretical Physics Department

Fermi National Accelerator Laboratory

P.O. Box 500, Batavia, IL 60510, USA

parke@fnal.gov

\begin{abstract}
The status and the most important issues in neutrino physics will be summarized as well as how the current, pressing questions will be addressed by future experiments.
\end{abstract}

Keywords:

\section{Neutrino Mass}

Probably the most fundamental question yet to be answer in neutrino physics is whether or not the massive neutrino is of Majorana or Dirac nature. If it is Majorana, then the massive neutrino has only two degrees of freedom, $\left(\nu_{L}\right.$ and $\left.\bar{\nu}_{R}\right)$, whereas if it is Dirac then there are four degrees of freedom $\left(\nu_{L}\right.$, $\nu_{R}, \bar{\nu}_{R}$ and $\left.\bar{\nu}_{L}\right)$. See Fig. 1 on how fermion masses are generated and Fig. 2 for the difference in the interactions of Majorana and Dirac neutrinos.

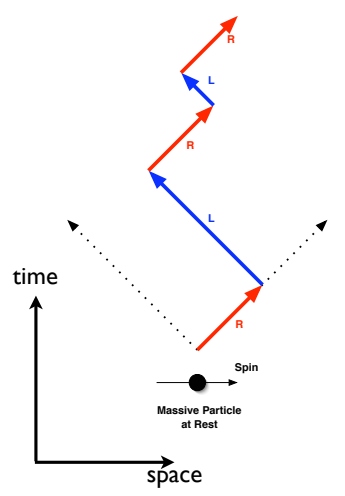

Figure 1: Fermion mass is a coupling between the right and left components of the Fermion field.

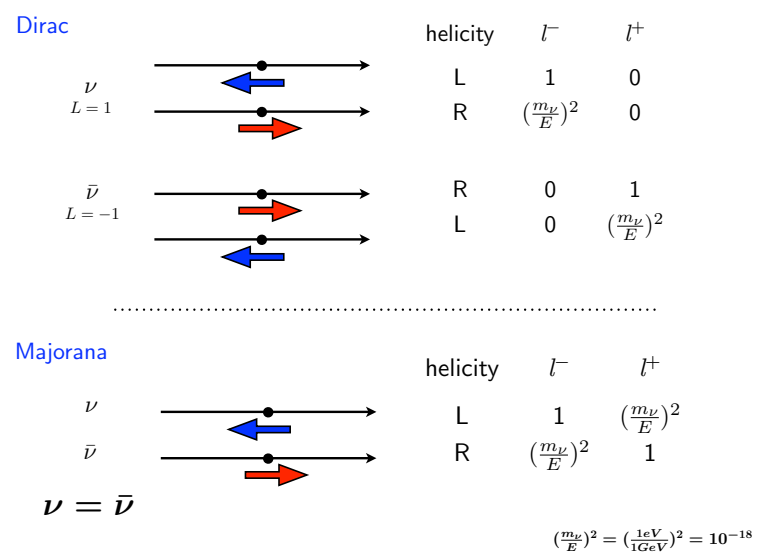

Figure 2: The difference between Majorana and Dirac neutrinos in a scattering experiment. Dirac neutrinos (as oppose to anti-neutrinos) only produce negatively charged leptons whereas a Majorana neutrino can produce charge leptons of either charge however the rate for the "wrong" charge is exceptionally small, $\left(m_{\nu} / E_{\nu}\right)^{2}$.

The Seesaw mechanism, which naturally splits a four component fermion into two massive Majorana fermions one exceptionally light and one very heavy, would provide a natural explanation of why the neutrinos masses are so much smaller than the other fermions of the standard model and also al- 
lows for the possibility of Leptogenesis. If the massive neutrinos were shown to be of Dirac nature, this would be a discovery of epic proportions in neutrino physics! An intense experimental effort is being devoted to observing the Majorana nature of the neutrino through neutrinoless double $\beta$-decay. See the session on neutrinoless double $\nu$-decay in this proceedings.

\section{Flavor content of the massive neutrinos}

Traditionally the neutrino mass eigenstates, $\nu_{1}$, $\nu_{2}$ and $\nu_{3}$, have been labelled such that:

$$
\begin{gathered}
\nu_{e} \text { component of } \nu_{1}>\nu_{e} \text { component of } \nu_{2} \\
>\quad \nu_{e} \text { component of } \nu_{3} .
\end{gathered}
$$

See Fig. 3 for the current information on the neutrino mass.

Therefore, the PMNS matrix elements for the $\nu_{e}$ flavor satisfy $\left|U_{e 1}\right|^{2}>\left|U_{e 2}\right|^{2}>\left|U_{e 3}\right|^{2}$ and since the only elements that have been measured by experiment so far are $\left|U_{e 2}\right|^{2},\left|U_{e 3}\right|^{2}$ and $\left|U_{\mu 3}\right|^{2}$, it is useful to choose a representation of this mixing matrix such that these elements are given approximately by the sine of a mixing angle,

$$
\begin{aligned}
\sin ^{2} \theta_{13} & \equiv\left|U_{e 3}\right|^{2}, \\
\sin ^{2} \theta_{12} & \equiv \frac{\left|U_{e 2}\right|^{2}}{\left(1-\left|U_{e 3}\right|^{2}\right)} \approx\left|U_{e 2}\right|^{2}, \\
\sin ^{2} \theta_{23} & \equiv \frac{\left|U_{\mu 3}\right|^{2}}{\left(1-\left|U_{e 3}\right|^{2}\right)} \approx\left|U_{\mu 3}\right|^{2}
\end{aligned}
$$

this is standard representation found in the PDG. The $\approx$ follows from the fact that we know that $\left|U_{e 3}\right|^{2}<<1$.
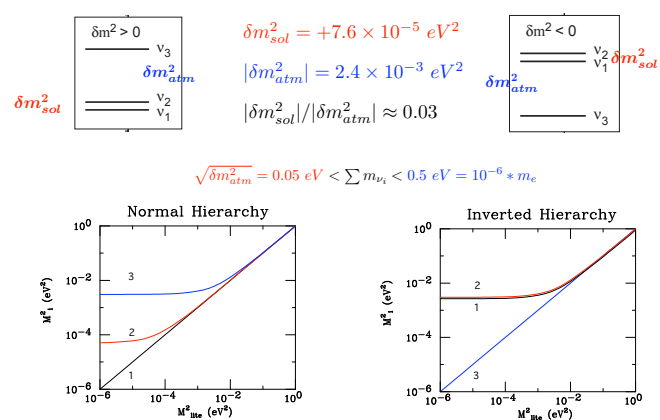

States 1 and 2 are $\nu_{e}$ rich.

Figure 3: What is known about the mass of the neutrino.

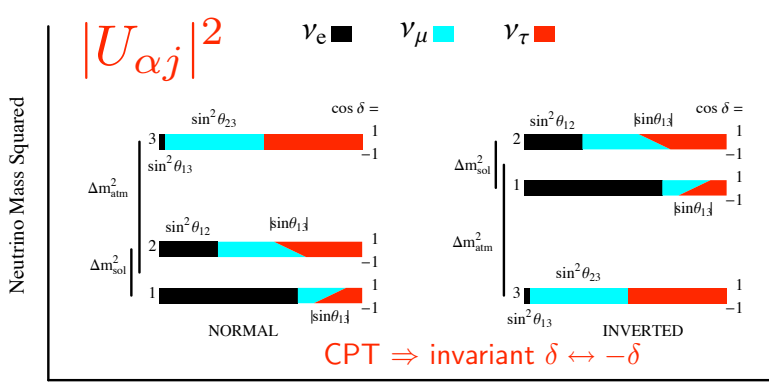

Fractional Flavor Content varying $\cos \delta$

Figure 4: The flavor content of the neutrino mass eigenstates[1]. The width of the lines is used to show how these fractions change as $\cos \delta_{C P}$ varies from -1 to +1 . Of course, this figure must be the same for neutrinos and antineutrinos if CPT is conserved.

At $2 \sigma$ we have the following experimental limits, see Fig. 4:

$$
\begin{aligned}
\sin ^{2} \theta_{13} & <0.04 \approx\left(\frac{\delta m_{21}^{2}}{\delta m_{31}^{2}}\right)^{1} \\
\left|\sin ^{2} \theta_{12}-\frac{1}{3}\right|<0.04 & \approx\left(\frac{\delta m_{21}^{2}}{\delta m_{31}^{2}}\right)^{1} \\
\left|\sin ^{2} \theta_{23}-\frac{1}{2}\right|<0.12 & \approx\left(\frac{\delta m_{21}^{2}}{\delta m_{31}^{2}}\right)^{0.6}
\end{aligned}
$$

where I have compared the size of the uncertainties to another small number appearing in the neutrino sector, the ratio of solar $\delta m^{2}, \delta m_{21}^{2}$, to the atmospheric $\delta m^{2}, \delta m_{31}^{2}$. The ratio $\delta m_{21}^{2} / \delta m_{31}^{2} \approx 0.03$.

The point in parameter space such that $\left(\sin ^{2} \theta_{13}, \sin ^{2} \theta_{12}, \sin ^{2} \theta_{23}\right)=\left(0, \frac{1}{3}, \frac{1}{2}\right)$ is known as Tri-Bimaximal mixing, [2] \& [3], and it is clear from Eq. (1) that nature has chosen a point close to tribimaximal mixing. Is this an accident or is it some kind of symmetry? To answer this question one needs to experimentally push on the limits in Eq. (1) to

$$
\left(\frac{\delta m_{21}^{2}}{\delta m_{31}^{2}}\right)^{n}
$$

where $n=2$ as the next round of experiments but eventually to high powers. It's possible the symmetry is not exact and there is some breaking about this tribimaximal point, then one would expect the deviations from tribimaximal to be related to one another by some small parameter. What is this small parameter and what are the relationships? In many ways this puzzle is much like determining 
the rules of chess when one has only been given a particular end game, see Fig. 5.

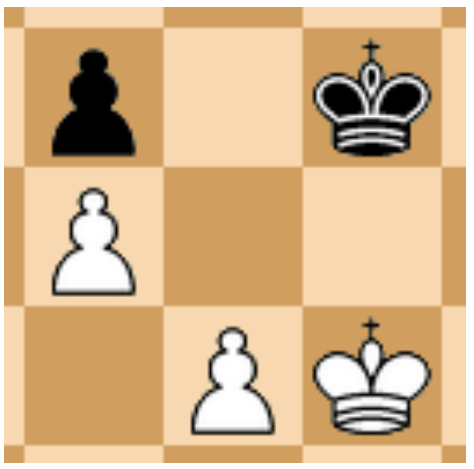

Figure 5: Part of an end game of chess. Determining how the neutrino mass matrix is organized is similar to determining the rules of chess given this end game. There are many piece (particles) that have already disappeared from the game!

\section{The Way Forward: $\sin ^{2} \theta_{13}$}

\subsection{Reactor Neutrinos}

Near the first oscillation minima for $\bar{\nu}_{e}$ disappearance from a reactor, the survival probability is given by

$$
\begin{aligned}
P\left(\bar{\nu}_{e} \rightarrow \bar{\nu}_{e}\right) \approx & 1-\sin ^{2} 2 \theta_{13} \sin ^{2}\left(\frac{\delta m_{e e}^{2} L}{4 E}\right) \\
& -\mathcal{O}\left(\frac{\delta m_{21}^{2} L}{4 E}\right)^{2}
\end{aligned}
$$

where $\delta m_{e e}^{2}$ is the $\nu_{e}$ weight average of $\left|\delta m_{31}^{2}\right|$ and $\left|\delta m_{32}^{2}\right|$, i.e. $\delta m_{e e}^{2} \equiv \cos ^{2} \theta_{12}\left|\delta m_{31}^{2}\right|+\sin ^{2} \theta_{12}\left|\delta m_{32}^{2}\right|$. Thus, such experiments are a great way to measure the parameter $\sin ^{2} \theta_{13}$ provide the systematic uncertainties are well controlled since $\sin ^{2} \theta_{13}$ is known to be small, $<0.03$. The reactor experiments Double Chooz, Daya Bay and Reno are currently under construction. See reactor talks in this proceedings.

\subsection{The Physics of Long Baseline: $\boldsymbol{\nu}_{\boldsymbol{\mu}} \rightarrow \boldsymbol{\nu}_{\boldsymbol{e}}$}

$\mathrm{T} 2 \mathrm{k}, \mathrm{NO} \nu, \mathrm{LBNE}, \beta$-Beams, Neutrino Factories etc will all search for non-zero $\sin ^{2} \theta_{13}$ via looking for $\nu_{\mu} \rightarrow \nu_{e}$ and it's related processes.

The amplitude for $\nu_{\mu} \rightarrow \nu_{e}$ can be simple written a sum of three amplitudes, one associated with each neutrino mass eigenstate,

$$
\begin{aligned}
U_{\mu 1}^{*} e^{-i m_{1}^{2} L / 2 E} U_{e 1} & +U_{\mu 2}^{*} e^{-i m_{2}^{2} L / 2 E} U_{e 2} \\
& +U_{\mu 3}^{*} e^{-i m_{3}^{2} L / 2 E} U_{e 3} .
\end{aligned}
$$

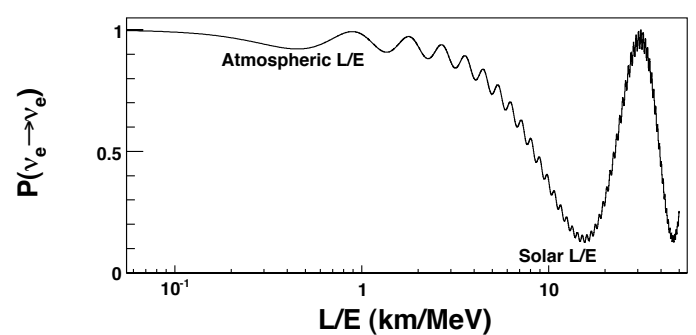

Figure 6: The survival probability for reactor neutrinos. The first oscillation minimum occurs at about $1.5 \mathrm{~km}$ from the source when integrated over the reactor neutrino spectra.

The first term can be eliminated using the unitarity of the MNS matrix and thus the appearance probability can be written as follows[4]

$$
P\left(\nu_{\mu} \rightarrow \nu_{e}\right) \approx\left|\sqrt{P_{a t m}} e^{-i\left(\Delta_{32}+\delta\right)}+\sqrt{P_{s o l}}\right|^{2} .
$$

$\Delta_{j k}$ is used as a shorthand for the the kinematic phase, $\delta m_{j k}^{2} L / 4 E$. As the notation suggests the amplitude $\sqrt{P_{a t m}}$ only depends on $\delta m_{31}^{2}$ and $\sqrt{P_{s o l}}$ only depends on $\delta m_{21}^{2}$. For propagation in the matter, these amplitudes are simple given by

$$
\begin{aligned}
& \sqrt{P_{a t m}}=\sin \theta_{23} \sin 2 \theta_{13} \frac{\sin \left(\Delta_{31}-a L\right)}{\left(\Delta_{31}-a L\right)} \Delta_{31} \\
& \sqrt{P_{\text {sol }}}=\cos \theta_{23} \sin 2 \theta_{12} \frac{\sin (a L)}{(a L)} \Delta_{21} . \quad \text { (4) }
\end{aligned}
$$

The matter potential is given by $a=G_{F} N_{e} / \sqrt{2} \approx$ $(4000 \mathrm{~km})^{-1}$ and the sign of $\Delta_{31}\left(\right.$ and $\Delta_{32}$ ) determines the hierarchy; normal $\Delta_{31}>0$ whereas inverted $\Delta_{31}<0$. When $a$ is set to zero one recovers the vacuum result. See Fig.7 [5].

For anti-neutrinos $a \rightarrow-a$ and $\delta \rightarrow-\delta$. Thus the phase between $\sqrt{P_{a t m}}$ and $\sqrt{P_{\text {sol }}}$ changes from $\left(\Delta_{32}+\delta\right)$ to $\left(\Delta_{32}-\delta\right)$. This changes the interference term from

$$
\Rightarrow \begin{aligned}
& 2 \sqrt{P_{\text {atm }}} \sqrt{P_{\text {sol }}} \cos \left(\Delta_{32}+\delta\right) \\
& \Rightarrow \quad 2 \sqrt{P_{\text {atm }}} \sqrt{P_{\text {sol }}} \cos \left(\Delta_{32}-\delta\right) .
\end{aligned}
$$

Expanding $\cos \left(\Delta_{32} \pm \delta\right)$, one has a $\mathrm{CP}$ conserving part

$$
2 \sqrt{P_{a t m}} \sqrt{P_{\text {sol }}} \cos \Delta_{32} \cos \delta
$$

and the $\mathrm{CP}$ violating part

$$
\mp 2 \sqrt{P_{\text {atm }}} \sqrt{P_{\text {sol }}} \sin \Delta_{32} \sin \delta .
$$


Therefore CP violation is maximum when $\Delta_{32}=$ $(2 n+1) \frac{\pi}{2}$ and grows as $\mathrm{n}$ grows. Notice also, that for this term to be non-zero the kinematical phase $\Delta_{32}$ cannot be $n \pi$. This is the neutrino counter part to the non-zero strong phase requirement for $\mathrm{CP}$ violation in the quark sector.

The asymmetry between $P\left(\nu_{\mu} \rightarrow \nu_{e}\right)$ and $P\left(\bar{\nu}_{\mu} \rightarrow \bar{\nu}_{e}\right)$ is a maximum when $\sqrt{P_{\text {atm }}}=\sqrt{P_{\text {sol }}}$. At the first oscillation maximum, $\Delta_{31}=\pi / 2$, this occurs when $\sin ^{2} 2 \theta_{13}=0.002$ in vacuum. For values of $\sin ^{2} 2 \theta_{13}<0.002$ the oscillation probabilities are dominated by $P_{\text {sol }}$ and thus observing the effects of non-zero $\sin ^{2} 2 \theta_{13}$ become increasing more challenging.
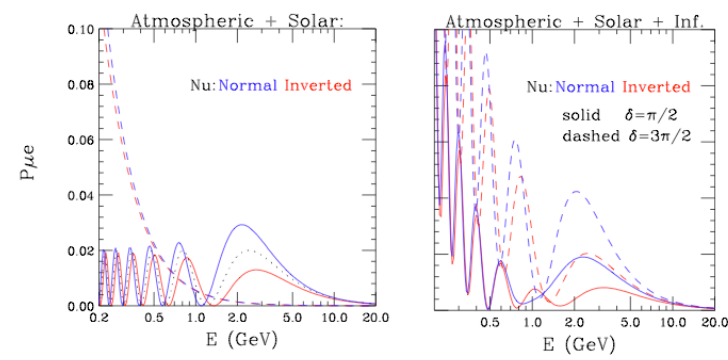

Figure 7: The left panel shows the two components $P_{\text {atm }}$ and $P_{\text {sol }}$ in matter for the normal and inverted hierarchies for $\sin ^{2} 2 \theta_{13}=0.04$ and a baseline of $1200 \mathrm{~km}$. The right panel shows the total probability including the interference term between the two components for various values of the $\mathrm{CP}$ phase $\delta$ for the neutrino. Notice that the coherent sum of two amplitudes shows a rich structure depending on the hierarchy and value of CP phase. These curves can also be interpreted as anti-neutrino probabilities if one interchanges the hierarchy AND the values of the CP phase.

\subsection{Comparing the results from Reactor and $L B L$ experiments}

A direct comparison can be made between the reactor and the long baseline experiments by comparing $\left.\left[P\left(\nu_{\mu} \rightarrow \nu_{e}\right)+P\left(\bar{\nu}_{\mu} \rightarrow \bar{\nu}_{e}\right)\right]\right|_{\Delta_{31}=\frac{\pi}{2}}$ from the long baseline experiments and $\left.1-P\left(\bar{\nu}_{e} \rightarrow \bar{\nu}_{e}\right)\right]\left.\right|_{\Delta_{31}=\frac{\pi}{2}}$ from the reactor experiments. Since

$$
\begin{aligned}
& {\left.\left[P\left(\nu_{\mu} \rightarrow \nu_{e}\right)+P\left(\bar{\nu}_{\mu} \rightarrow \bar{\nu}_{e}\right)\right]\right|_{\Delta_{31}=\frac{\pi}{2}}=} \\
& 2 \sin ^{2} \theta_{23} \sin ^{2} 2 \theta_{13}+\mathcal{O}[(a L) \sin \delta]
\end{aligned}
$$

and

$$
\left.1-P\left(\bar{\nu}_{e} \rightarrow \bar{\nu}_{e}\right)\right]\left.\right|_{\Delta_{31}=\frac{\pi}{2}}=\sin ^{2} 2 \theta_{13}
$$

then this comparison is a way to measure by how much $\sin ^{2} \theta_{23}$ differs from $\frac{1}{2}$ including the sign, so is a way to determine the quadrant of $\theta_{23}$. Fig. 8

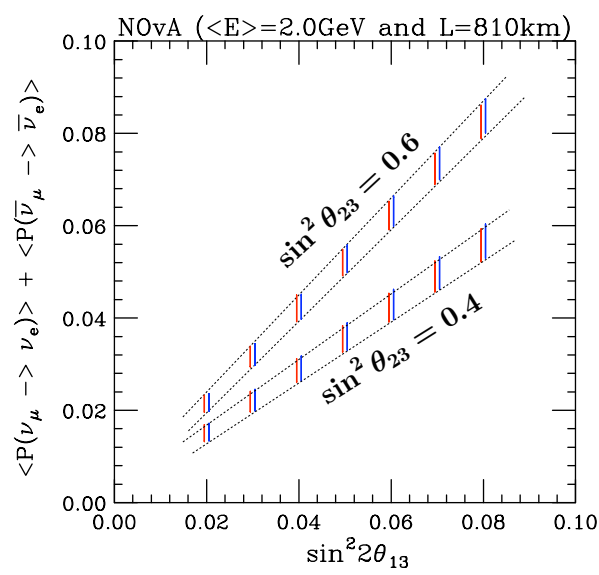

Figure 8: The sum of the $\nu_{e}$ and $\bar{\nu}_{e}$ appearance probabilities for the NOvA experiment verses $\sin ^{2} 2 \theta_{13}$. The regions between the dotted lines give the allowed ranges for $\sin ^{2} 2 \theta_{23}=0.6$ and $\sin ^{2} 2 \theta_{23}=0.4$ which are well separated for large values of $\sin ^{2} 2 \theta_{13}$ and some what insenitive to the mass hierarchy.

shows that this comparison works reasonable well even when the long baseline experiment is not exactly at the vacuum oscillation extremuum.

\section{Beyond Superbeams: $\beta$-Beams and $\mu$ - Storage Rings}

To access the physics for small values of $\sin ^{2} 2 \theta_{13}$, approximately $<0.01$, one requires neutrino beams with small $(<<1 \%)$ contamination of the appearance flavor of neutrinos. Two possibilities are under intense investigation:

(I) $\beta$-Beams, where the neutrinos are produced by the $\beta$-decay of a nucleus,

$$
N \rightarrow N^{\prime}+e-\left(e^{+}\right)+\bar{\nu}_{e}\left(\nu_{e}\right) .
$$

Two pairs of nuclei are under investigation ${ }^{6} \mathrm{He}$ and ${ }^{18} \mathrm{Ne}$ as well as ${ }^{8} \mathrm{~B}$ and ${ }^{8} \mathrm{Li}$. These ions are accelerated to high energy, $\gamma \sim O(100)$ ), with a resulting neutrino energy of order of $1 \mathrm{GeV}$ or less and therefore correspondingly baselines of $<1000 \mathrm{~km}$. The detectors for this setup must be optimized to separate electron events from muon events. Neutrino beams produced by this technique are better suited to investigating $\mathrm{CP}$ violation than the neutrino mass hierarchy, see E. Wildner this proceedings.

(II) Another possibility is to produce the neutrinos from a muon storage ring, this option has been 
called the Neutrino Factory. In this option the neutrinos are produced via

$$
\begin{gathered}
\mu^{+} \rightarrow e^{+}+\nu_{e}+\bar{\nu}_{\mu} \\
\text { or } \quad \mu^{-} \rightarrow e^{-}+\bar{\nu}_{e}+\nu_{\mu} .
\end{gathered}
$$

So here the detector must be able to determine the charge of the muon events with very small misassignments. This is not so easy at small energy thus the neutrinos from this option are usually assumed to be $10 \mathrm{GeV}$ or so, therefore the baselines are correspondingly longer. Neutrino beams produced by this technique are naturally better suited to determining the neutrino mass hierarchy than $\mathrm{CP}$ violation, see K. Long this proceedings.

\section{Anomalies}

\section{1. $L S N D / \operatorname{miniBooNE}$}

The hypothesis that LSND anomaly is due to neutrino oscillations has been tested by miniBooNE experiment. In the neutrino channel miniBooNE does not support this hypothesis whereas in the anti-neutrino miniBooNE does provide some supporting evidence for the oscillation interpretation of the LSND anomaly. More information on this topic can be found in Van der Water's and Karagiorgi's talks at this conference.

\section{2. $M I N O S$}

The MINOS experiment has compared the oscillation parameters, $\left(\sin ^{2} 2 \theta, \delta m^{2}\right)$, for neutrinos and anti-neutrinos. At this time there is some tension between these two results. Further data was presented at this conference, increasing the tension, see the talk by Vahle. If this disappearance experiment was performed in the vacuum then the results for neutrino running and anti-neutrino running should be identical due to CPT invariance. However, since these experiments are performed in earth matter there is the possibility that some new interaction with the earth matter is responsible for the difference between neutrino and anti-neutrinos. Further data from MINOS and the experiments $\mathrm{T} 2 \mathrm{~K}$ and $\mathrm{NO} \nu \mathrm{A}$ will further illuminate this topic.

\section{Conclusions}

Since the discovery of neutrino flavor transitions by the SuperKamiokande experiment in 1998, which demonstrates that neutrinos change and hence their clocks tick, i.e. they are not traveling at the speed of light and hence are not massless, the field of neutrino physics has made remarkable progress in untangling the nature of the neutrino. However, there are still many important questions still to answer:

- Confirm or refute the Majorana nature of the neutrino.

- Are neutrinos quasi-degenerate or hierarchical in mass?

- If hierarchical what is the mass of the lighest neutrino? What sets this scale?

- Is the spectrum Normal or Inverted?

- How close is the mixing matrix to Tri-BiMaximal mixing?

- What is the size and sign of CPV ?

- Are there light sterile neutrinos?

- Where are the surprises?

We all have prejudices about how nature has organized the neutrino sector, she has surprises in store for us. We need to find them.

In 1966 Isaac Asimov wrote a book titled "The Neutrino: the ghost particle of the atom" and in this book he concluded that

\section{"And yet the nothing-particle is not a nothing at all."}

This statement is more true than Asimov could have known. Yet, I think, even today, we have only just glimpsed the wonders of the mysterious neutrino !!!

\section{References}

[1] O. Mena and S. J. Parke, Phys. Rev. D 69, 117301 (2004) [arXiv:hep-ph/0312131].

[2] L. Wolfenstein, Phys. Rev. D 18, 958 (1978).

[3] P. F. Harrison, D. H. Perkins and W. G. Scott, Phys. Lett. B 530, 167 (2002) [arXiv:hep-ph/0202074].

[4] A. Cervera, A. Donini, M. B. Gavela, J. J. Gomez Cadenas, P. Hernandez, O. Mena and S. Rigolin, Nucl. Phys. B 579, 17 (2000) [Erratum-ibid. B 593, 731 (2001)] [arXiv:hep-ph/0002108].

[5] H. Nunokawa, S. J. Parke and J. W. F. Valle, Prog. Part. Nucl. Phys. 60, 338 (2008) [arXiv:0710.0554 [hep$\mathrm{ph}]]$. 